\title{
Evaluation of the Microcult system for isolating and identifying Neisseria gonorrhoeae
}

\author{
R. J. Williams, C. S. RATNATUNGA, J. M. T. HAMilton-Miller, AND \\ W. BRUMFITT
}

From the Departments of Medical Microbiology and Venereology, Royal Free Hospital, London $N W 3, U K$

SUMMARY Specimens from 95 patients attending a venereal diseases clinic were examined for gonococci by three methods - a conventional culture technique using modified Thayer-Martin medium, microscopy of a Gram-stained direct smear, and the Microcult system. For $56 \%$ of the specimens the results by all three methods agreed. Assuming the results obtained by culture on Thayer-Martin medium to be correct, the largest source of error was due to false-positive results: microscopy gave 26 and Microcult gave 15 such results. False-negative results were less common: Microcult gave 14, microscopy six. Microcult gave positive results more quickly than the conventional Thayer-Martin cultural method, but the gonococci were difficult to isolate by subculture from the Microcult culture pads. The Microcult medium was not absolutely specific for Neisseria gonorrhoeae. Nevertheless, the Microcult test may well prove to be a useful adjunct to the diagnosis of gonorrhoea, especially when laboratory facilities are not readily available.

A presumptive diagnosis of gonorrhoea is often made from the clinical history and the examination of a Gram-stained smear taken directly from the patient. However, confirmation by culture and identification of Neisseria gonorrhoeae is essential whenever practicable. Because $N$. gonorrhoeae is a fastidious organism complex and selective media such as that of Thayer and Martin $(1964,1966)$ or one of its many modifications are needed for its primary isolation.

Transporting specimens from clinic to laboratory is often a problem. $N$. gonorrhoeae dies rapidly on dry swabs and even the use of charcoal swabs and appropriate transport media (Stuart, 1959; Amies, 1967; Wilkinson et al., 1972) has not fully solved this difficulty. In the most favourable circumstances recovery of gonococci is much reduced when there is more than 24 hours' delay in transit. Efforts have been made to combine transport and culture media in an easily transportable container, analogous to dip slides used for urine. This avoids the need to plateout the specimen on arrival. Microcult (Ames) is one such method, but its reliability is still under investigation.

In this study, firstly, we compared the Microcult

Received for publication 8 August 1977 method with conventional microscopy and culture methods, secondly, we assessed its overall performance, and, thirdly, we determined whether it enabled a tentative diagnosis of gonorrhoea to be made more rapidly.

\section{Material and methods}

A Microcult kit (Miles Laboratories Ltd) consists of a plastic slide on which are two areas of desiccated modified Thayer-Martin medium, each about $4 \mathrm{~cm}^{2}$ in size. This is enclosed within a foil pouch that is used for both storage and incubation. Immediately before use the pouch is opened, the slide removed, and the medium rehydrated with sterile distilled water (six drops for each area). Both areas are then inoculated with material from the patient, the slide is replaced inside the pouch, and a $\mathrm{CO}_{2}$-generating tablet is added; the pouch is closed by folding over the foil and incubated at $37^{\circ} \mathrm{C}$. After 24 hours' incubation a strip impregnated with oxidase reagent (tetramethyl-p-phenylenediamine dihydrochloride) is pressed on to one of the inoculated areas for 20 seconds. If $N$. gonorrhoeae is present purple spots appear on the matrix within 10 seconds of removing the strip. If the test is negative the slide is returned to 
the pouch and reincubated for a further 24 hours, and the test is repeated on the second area of medium.

Thayer-Martin agar (TMA) was made up in the microbiology department of the Royal Free Hospital by combining 'GC agar', haemoglobin, and Supplement A (all Difco Bacto products), according to the manufacturer's instructions, and adding vancomycin ( $3 \mu \mathrm{g} / \mathrm{ml})$, colistimethate $(7 \cdot 5 \mu \mathrm{g} / \mathrm{ml})$, trimethoprim lactate $(3 \mu \mathrm{g} / \mathrm{ml})$, and pimaricin $(20 \mu \mathrm{g} / \mathrm{ml})$.

Specimens were taken from patients attending the department of venereology by means of a cottonwool charcoal swab (Exogen Ltd) or a smooth sterile wire loop. Three separate specimens were taken. The first was used to inoculate Stuart's medium, the second was used to make a smear for microscopical examination, and the third was used to inoculate the Microcult kit. The order in which the specimens were taken for transport and for Microcult was alternated.

The direct smears were stained in the clinic by Gram's method and examined immediately by an experienced member of the clinic staff. The swab in transport medium was sent to the microbiology department by the usual delivery system, arriving within a maximum of four hours. The swabs were cultured on TMA and incubated at $37^{\circ} \mathrm{C}$ in a $\mathrm{CO}_{2}$ incubator (Gallenkamp; atmosphere $90 \%$ air $+10 \%$ $\mathrm{CO}_{2}$ ). Plates were examined after 24 and 48 hours, colonies were tested for oxidase reaction, and Gram staining and standard sugar fermentation tests were performed.

Microcult kits were inoculated, incubated, and tested with oxidase strips as described above. Subculture from the test areas was attempted, as follows: a loopful of distilled water was placed at the edge of the culture area, which was then scraped gently with a loop to remove some of the matrix in which the bacteria grow; the loopful was then plated out on to TMA and incubated as already described.

In keeping with conventional practice the finding on microscopy of intracellular Gram-negative diplococci was considered a positive result. TMA cultures were considered positive when the organisms isolated were Gram-negative, oxidase-positive diplococci that fermented glucose but not maltose. A positive Microcult result was recorded when purple spots appeared after the test strip had been pressed on the culture area. An overall pinkish tinge, which sometimes developed several minutes after testing, was reported as negative.

As a standard of comparison we assumed that the results of culture on TMA gave the 'correct' answer. This enabled us to tabulate the performance of the Microcult system and of microscopy in terms of true positive (TP), true negative (TN), false positive (FP), and false negative (FN). These data can be used to calculate the specificity, sensitivity, and predictive values for a positive and for a negative test (Krieg $e \overrightarrow{\bar{t}}$ $a l .$, 1975). These terms are defined and calculated ino the following manner. Sensitivity is the percentage of positive results in patients with the disease $\left(=\left(\mathrm{TP} / \frac{\mathrm{o}}{\mathrm{C}}\right.\right.$ $(T P+F N)) \times 100)$. Specificity is the percentage of negative results among people who do not have theos disease $(=(\mathrm{TN} /(\mathrm{TN}+\mathrm{FP})) \times 100)$. The predictives values define the percentage of results that are true that is, $(\mathrm{TP} \times 100) /(\mathrm{TP}+\mathrm{FP})$ for positives and $(\mathrm{TN} \times \overrightarrow{\vec{\omega}}$ $100) /(T N+F N)$ for negatives.

\section{Results}

AGREEMENT BETWEEN METHODS

Results using all three methods were obtained on 950 specimens-64 urethral, 19 cervical, and 12 rectal-은 obtained from 66 patients ( 45 men, 21 women). Out of the specimens 25 were positive and 29 weres negative by all three tests. The remaining 41 fell into various categories (Table 1).

Table 1 Results of testing 95 specimens by Microcult, standard culture, and microscopy

\begin{tabular}{|c|c|c|c|}
\hline \multicolumn{3}{|l|}{ Method } & \multirow[b]{2}{*}{ No. $(\%)$} \\
\hline Microscopy & Microcult & $T M A$ & \\
\hline$\div$ & & & $25(26)$ \\
\hline- & - & - & $29(30)$ \\
\hline- & - & $T$ & $5(5 \cdot 3)$ \\
\hline- & $\because$ & - & $-(-)$ \\
\hline+ & - & - & $11(11 \cdot 6)$ \\
\hline- & $T$ & $\therefore$ & $1(1 \cdot 1)$ \\
\hline+ & - & + & $9(9 \cdot 5)$ \\
\hline+ & + & - & $15(15 \cdot 8)$ \\
\hline
\end{tabular}

TMA $=$ Thayer-Martin agar $+=$ positive result $;-=$ negative result.

SPECIFICITY AND SENSITIVITY

Results are shown in Table 2. The Microcult systemo gave $26 \mathrm{TP}, 40 \mathrm{TN}, 15 \mathrm{FP}$, and $14 \mathrm{FN}$. Microscopy gave fewer FN results (6) and more FP (26) thano Microcult.

Table 2 Comparison of performance of Microcult method and microscopy with culture on Thayer-Martin agar

\begin{tabular}{|c|c|c|c|c|}
\hline & \multirow{2}{*}{$\begin{array}{l}\text { Specificity } \\
\%\end{array}$} & \multirow{2}{*}{$\begin{array}{l}\text { Sensitivity } \\
\%\end{array}$} & \multicolumn{2}{|c|}{ Predictive value $(\%)$ for: } \\
\hline & & & + Result & - Result \\
\hline Microcult & $72 \cdot 7(100)^{*}$ & $65(75 \cdot 3)^{*}$ & $63.4(100)^{*}$ & $74 \cdot 1(76 \cdot 3)^{*}$ \\
\hline Microscopy & $53 \cdot 7$ & 85 & $56 \cdot 7$ & 82.9 \\
\hline
\end{tabular}

* Figures in parenthesis are results obtained when stricter criteria are applied-that is, when both TMA culture and microscopy agreed.

When the criterion for a 'correct' answer was stricter-namely, when both TMA culture and 
microscopy agreed -63 specimens were available for analysis. Microcult gave no FP and nine FN, resulting in a much improved specificity $(100 \%)$ and predictive value for a positive result $(100 \%)$ and marginally better sensitivity $(73.5 \%)$ and predictive value for a negative result $(75 \cdot 7 \%)$ (Table 2$)$.

Table 1 shows that 15 specimens gave FP results by both Gram-film examination and Microcult. There is no record that any of the patients from whom these specimens came had any other epidemiological evidence of gonorrhoea.

\section{RAPIDITY OF CULTURAL METHODS}

The manufacturer's literature states that the Microcult system produces positive results more quickly than by conventional culture methods. Our results bore this out. A total of 41 Microcult tests were positive and in $29(70.7 \%)$ of them the result was positive by 24 hours. Of the 40 positive TMA tests only $16(40 \%)$ were positive by 24 hours. In 26 cases both TMA and Microcult were positive: in 10 $(38.5 \%)$ of them Microcult gave a positive result earlier, while in only $4(15.4 \%)$ was the reverse true.

\section{Discussion}

Several systems have been developed over the last 10 years to try to overcome the problems of transport and culture of the fastidious gonococcus. The performance of methods such as Transgrow (Martin and Lester, 1971; Brown, 1974), Gonogrow (Chen et al., 1974), Gonocult (Karnes et al., 1975), and the Jembec-Neigon system (Jephcott et al., 1976) have not established clearly the superiority either of one over another or of any one over existing conventional methods. In addition a serious disadvantage of all these kits is their short shelf life (about two months). The Microcult system is still under investigation but its shelf life seems to be virtually infinite, presumably because the medium is stored in a desiccated form.

Some of the apparent FP results obtained with Microcult may be explained if the parallel swab for culture on TMA, which might have yielded a positive result, was delayed in transit so that any gonococci on it had died. Precisely the same argument applies to the results obtained by microscopy. A major advantage of the Microcult system is that it is inoculated directly, so that transport problems do not arise.

Other FP results can be accounted for by the growth of oxidase-positive organisms other than gonococci on the culture pads. The TMA of the Microcult system is designed specifically to prevent the growth of bacteria other than neisseriae. However, when we inoculated a strain of Pseudomonas aeruginosa on to the pads it grew well; the same applied to a strain of $N$. meningitidis and to commensal neisseriae inoculated from broth cultures. Microcult is thus clearly totally unsuitable for examining throat swabs for gonococci, and $N$. meningitidis has occasionally been isolated from the genital area (Lewis and Alexander, 1974). Furthermore, since it is becoming apparent that oxidasepositive Gram-negative bacteria of genera other than Neisseria spp may inhabit the genital tract (Platt and Snell, 1976) it would seem mandatory to isolate and identify as $N$. gonorrhoeae all oxidase-positive organisms growing in the Microcult system before reporting to the venereologist. Thus the kit is unsuitable for the microbiological diagnosis of gonorrhoea by those without access to a laboratory. Nevertheless, it may well be a useful transport medium.

Owing to the small volume of material sampled by the loop or swab, and thus possible sampling error, consecutive swabs may give different results, especially if the gonococci are present in only relatively small numbers. Because of this both FP and FN results will be generated (Dans, 1976).

Taking all these points into account the performance of Microcult was creditable, especially when the stricter of the two criteria for a 'correct' answer was taken (that is, when both TMA culture and microscopy agreed).

Clearly, also, a positive finding on Microcult is of greater significance than a negative one, whereas with microscopy the reverse is true.

Therefore we agree with the preliminary report of Willcox and John (1976) that Microcult could be a useful adjunct to the diagnosis of gonorrhoea provided certain limitations are recognised. Its compactness and long shelf life are strongly in its favour, as is the greater speed with which it gives positive results. Against this must be set the fact that subculture is essential to obtain viable colonies for identification purposes. Despite following with care the manufacturer's instructions for subculturing organisms off the pad we could not isolate gonococci regularly from known positive specimens. This is a serious disadvantage, since sensitivity testing is impossible if the organism cannot be isolated from the pads. Sensitivity testing must now be considered mandatory in view of recent reports of penicillinresistant strains, whether $\beta$-lactamase-producing (Percival et al., 1976) or not (Wilson et al., 1976).

\section{References}

Amies, C. R. (1967). A modified formula for the preparation of Stuart's transport medium. Canadian Journal of Public Health, 58, 296-300.

Brown, J. D. (1974). Modified transport and growth medium for the cultivation of Neisseria gonorrhoeae. British Journal of Veneral Diseases, 50, 199-201. 
Chen, N. C., Hipp, S. S., Lawton, W. D., and Gaafar, H. A. (1974). Gonogrow, an improved, selective medium for the isolation of Neisseria gonorrhoeae. Health Laboratory Sciences, 11, 173-177.

Dans, P. E. (1976). Diagnostic tests for sexually transmitted disease (letter). Journal of the American Medical Association, 235, 2583.

Jephcott, A. E., Bhattacharyya, M.N., and Jackson, D. H. (1976). Improved transport and culture system for the rapid diagnosis of gonorrhoea. British Journal of Veneral Diseases, 52, 250-252.

Karnes, D. D., Murray, R., Caruso, J., and Gladdman, J. M. (1975). Clinical evaluation of a new system for the detection of suspected gonorrhoeal infection. Current Therapeutic Research, 17, 578-584.

Krieg, A. F., Gambino, R., and Galen, R. S. (1975). Why are clinical laboratory tests performed? When are they valid? Journal of the American Medical Association, 233, 76-78.

Lewis, J. F., and Alexander, J. J. (1974). Isolation of Neisseria meningitidis from the vagina and cervix. American Journal of Clinical Pathology, 61, 216-217.

Martin, J. E., and Lester, A. (1971). Transgrow, a medium for transport and growth of Neisseria gonorrhoeae and Neisseria meningitidis. Health Services and Mental Health Administration Health Reports, 86, 30-33.

Percival, A., Rowlands, J., Corkill, J. E., Alergant, C. D., Arya, O. P., Rees, E., and Annels, E. H. (1976).

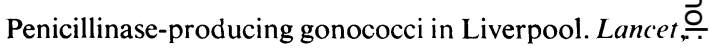
2, 1379-1382.

Platt, D. J., and Snell, J. J. S. (1976). Isolation of oxidase positive Gram-negative cocci not belonging to genuse Neisseria from the urogenital tract. Journal of Clinica $\underline{\underline{\underline{p}}}$ Pathology, 29, 537-542.

Stuart, R. D. (1959). Transport medium for specimens in public health bacteriology. Public Health Reports, 74 $431-438$.

Thayer, J. D., and Martin, J. E., Jr. (1964). A selective medium for the cultivation of Neisseria gonorrhoeae andNeisseria meningitidis. Public Health Reports, 79, 49. 57.

Thayer, J. D., and Martin, J. E. (1966). Improved mediunos selective for cultivation of $N$. gonorrhoeae and $N \omega$ meningitidis. Public Health Reports, 81, 559-562.

Wilkinson, A. E., Turner, G. C., and Rycroft, J. A. (1972)i The bacterial diagnosis of gonorrhoea. In Laboratorye Diagnosis of Veneral Disease (Public Health Laboratoryo Service Monographs, No. 1) edited by A. E. Wilkinson, et al., pp. 35-39. HMSO, London.

Willcox, R. R., and John, J. (1976). Simplified method foo the cultural diagnosis of gonorrhoea. British Journal of Veneral Diseases, 52, 256.

Wilson, C., Rose, D. L., and Tramont, E. C. (1976) Increased antibiotic resistance of Neisseria gonorrhoeac.00 in Korea. Antimicrobial Agents and Chemotherapy, 9.8 716-718. 